\title{
Assessment of myocardial viability with delayed-enhancement MRI in coronary artery disease: A correlative study with coronary artery stenosis using digital subtraction angiography
}

\author{
XINXIANG ZHAO ${ }^{1}$, YANGLIN ZHANG ${ }^{1}$, YONG SUN $^{1}$, LIN SUN $^{2}$ and RENHUI CAI ${ }^{1}$ \\ Departments of ${ }^{1}$ Radiology and ${ }^{2}$ Cardiology, The Second Affiliated Hospital, \\ Kunming Medical University, Kunming, Yunnan 650101, P.R. China
}

Received August 26, 2014; Accepted April 13, 2016

DOI: $10.3892 / \mathrm{etm} .2016 .3590$

\begin{abstract}
The aim of the present study was to investigate the correlation between the degree of coronary artery stenosis determined by digital subtraction angiography (DSA) and infarcted segments detected by delayed enhancement magnetic resonance imaging (DE-MRI). DE-MRI and DSA were performed in 40 patients with coronary artery disease. The number of myocardial segments with infarction, the transmural extent of myocardial infarction, score of myocardial infarction by MRI, degree of coronary artery stenosis and Gensini score of the coronary artery were assessed. The correlation was analyzed using Spearman's rank correlation test. Among the 40 patients, 126 infarcted myocardial segments with a total score of 307 were found by DE-MRI; the total Gensini score for coronary artery stenosis was 587 . It was observed that $81.74 \%$ of the infarcted segments were at sites with $>50 \%$ coronary artery stenosis. The correlation coefficient between the Gensini score and myocardial infarction score was 0.786 $(\mathrm{P}<0.001)$, indicating a good correlation. However, $18.26 \%$ of myocardial infarction segments were found in patients with slight coronary artery stenosis $(\leq 25 \%)$. A correlation was identified between DSA detected coronary artery stenosis and infarcted segments detected by DE-MRI; a higher transmural extent of myocardial infarction correlated with more severe stenosis of the coronary artery. The combined use of the two tools may facilitate accurate diagnosis.
\end{abstract}

\section{Introduction}

Digital subtraction angiography (DSA) allows the degree of patency or stenosis of blood vessels to be rapidly quantified

Correspondence to: Dr Xinxiang Zhao, Department of Radiology, The Second Affiliated Hospital, Kunming Medical University, 374 Wuhua Burma Road, Kunming, Yunnan 650101, P.R. China E-mail: 422562482@qq.com

Key words: myocardial infarction, coronary artery stenosis, magnetic resonance imaging, delayed enhancement and is now a routine clinical imaging modality; DSA of the coronary artery is one of its main applications (1). Delayed enhancement magnetic resonance imaging (DE-MRI) enables the accurate detection and visualization of myocardial scar tissue. When compared with single-photon emission computed tomography (SPECT), DE-MRI has been found to be more likely to detect small myocardial scars as it has higher sensitivity (2). DE-MRI has been reported to be capable of detecting lesions with a mass of $\sim 2 \mathrm{~g}$ while SPECT could only detect those with a mass of $\geq 10 \mathrm{~g}$ (3). These missed small infarcts could potentially be more dangerous than larger infarcts $(4,5)$.

Although DE-MRI has certain advantages in the detection of myocardial infarction, particularly for small infarcts and subendocardial infarcts, its clinical application remains rare. Therefore, the present study aimed to investigate the correlation between the transmural extent of infarction in myocardial segments detected by DE-MRI and the degree of coronary artery stenosis detected by DSA.

\section{Materials and methods}

Patients. This prospective study involved 40 patients with a history of coronary artery disease (CAD) and with myocardial infarction segments determined by DE-MRI. The study group included 32 men and 8 women, whose ages ranged from 26 to 72 years (mean, 53.3 \pm 11.6 years). Patients were recruited from the Second Affiliated Hospital of Kunming Medical University (Kunming, China), between June 2009 and November 2013. The study was approved by the ethics committee of rhe Second Affiliated Hospital of Kunming Medical University and written informed consent was obtained from each patient or their guardians

DE-MRI and coronary DSA. MRI investigations were performed using a 1.5T scanner (Siemens Sonata; Siemens AG, Munich, Germany). Imaging parameters were as follows: repetition time ( $\mathrm{msec}) /$ echo time $(\mathrm{msec}) /$ inversion time (msec), 700/4.18/250-300; matrix, 256x64; flip angle, 25 ; inversion pulse, section thickness, $6 \mathrm{~mm}$; field of view, 280x75 mm; bandwidth, $130 \mathrm{~Hz}$.

Images were obtained in the following orientations: 4-chamber, 2-chamber-long axis and a short axis stack covering 
the entirety of the left ventricle from the base to the apex. Gadolinium-diethylenetriamine pentaacetic acid (Guangdong Consun Pharmaceutical Group, Guangzhou, China) was injected twice, with a total dose of $0.2 \mathrm{mmol} / \mathrm{kg}$ administered intravenously. Following a 5-30 min delay, images were obtained under breath-holding conditions. Coronary angiography was performed with an Axiom Artis dTA angiography system (Siemens AG).

Image analysis. On the images obtained by DE-MRI, the left ventricle was divided into 17 segments according to the American Heart Association standards (6). The transmural extent of myocardial infarction in each segment was graded from 0 to IV: 0 , normal; I, $<25 \%$ transmural extent, scored 1 ; II, 26-50\%, scored 2; III, 51-75\%, scored 3; IV, 76-100\%, scored 4 . These tests were carried out by two radiologists using a blinding method.

DSA coronary artery analysis. The Gensini scoring system that was used has been described and validated previously (7). The severity of coronary artery stenosis was given a total score of between 1 and 32, as follows: <25\%, scored 1; 26-50\%, scored $2 ; 51-75 \%$, scored $4 ; 76-90 \%$, scored $8 ; 91-99 \%$, scored 16; $100 \%$, scored 32. All scores were confirmed together by one senior interventional cardiologist and one interventional radiologist.

Statistical analysis. Statistical analysis was performed using SPSS software, version 13.0 (SPSS, Inc., Chicago, IL, USA). Correlation between coronary artery Gensini scores and myocardial infarction segmental scores was analyzed by Spearman's rank correlation analysis. The Kendall rank correlation method was used to analyze the correlation between the extent of coronary artery stenosis as determined by DSA and the transmural extent of myocardial infarction.

\section{Results}

DSA coronary angiography. Analysis of the DSA coronary angiography images indicated that there were 37 cases of coronary artery stenosis among the 40 patients: There was single-vessel disease in 12 cases, double-vessel disease in 10 cases, and triple or more-vessel disease in 15 cases. In total, 87 vessels containing angiostenosis were identified and 3 cases showed no signs of abnormality. DSA images from a patient with coronary artery stenosis are shown in Fig. 1A and $\mathrm{B}$, and from a patient with no signs of stenosis are shown in Fig. 2A and B.

DE-MRI detection. The DE-MRI results revealed 126 segments with myocardial infarction and delayed enhancement in the 40 cases (Figs. 1C and 2C). Among these segments, the percentages of grade I, II, III and IV were $31.75 \%(n=40)$, $19.05 \%(n=24), 22.22 \%(n=28)$ and $26.98 \%(n=34)$.

Correlation analysis between coronary Gensini score and segmental myocardial infarction detected by DE-MRI. The total coronary Gensini score was 587, while the total DE-MRI score was 308. For the Spearman's rank correlation, the correlation coefficient, $\mathrm{r}_{\mathrm{s}}=0.786(\mathrm{P}<0.001)$, indicating a good correlation between DE-MRI-detected myocardial infarction and the extent of coronary artery stenosis. The Kendall rank correlation coefficient was $0.405(\mathrm{P}<0.001)$.

Table I shows the extent of coronary artery stenosis as determined by DSA and the transmural extent of myocardial infarction in each segment as detected by DE-MRI.

\section{Discussion}

DSA is the gold standard for the imaging of coronary arteries. However, in the clinic, knowing only the extent of coronary stenosis is insufficient to assess the coronary arteries. Patients with coronary artery disease and myocardial infarction can exhibit myocardium with a variety of functional states including stunned, hibernating and nonviable (8). The first two states are reversible damage, and the myocardium is viable, while the last state is irreversible. The identification of viable myocardium is important for the guidance of treatment and the judgment of prognosis. Clinical studies have shown an excellent correspondence between infarction as revealed by DE-MRI and histopathology (9-12). Therefore, the ability of DE-MRI to accurate identify the viable myocardium suggests its potential as a diagnostic procedure to be combined with DSA.

In the 40 patients involved in the present study, the Spearman's rank correlation coefficient between DSA coronary artery Gensini score and DE-MRI myocardial infarction score was $0.786(\mathrm{P}<0.001)$, indicating a positive correlation between coronary stenosis and myocardial infarction. A higher transmural extent of myocardial infarction was found to correlate with more severe stenosis of the coronary artery (Table I). Furthermore, $81.74 \%$ of the segments with myocardial infarction were located in areas of vascular blood flow with a degree of coronary artery stenosis of $\geq 50 \%$. Among the segments in which the transmural extent of myocardial infarction was $>75 \%$, and $64.71 \%$ of the segments were present in areas of blood supply that had $>90 \%$ coronary stenosis.

The identification of segments and sites affected by myocardial infarction, and the transmural extent of myocardial infarction by DE-MRI can be used to supplement examination of coronary arteries by DSA, and may guide the treatment and prognosis of patients. A study of chronic myocardial recovery capability found that blood supply reconstruction is inversely proportional to the transmural extent of myocardial infarction; in segments with $>50 \%$ transmural extent of infarction, the cardiac function was unlikely to be completely recovered (13). Therefore, the assessment of the transmural extent of myocardial infarction in myocardial segments by DE-MRI is a potential option in clinical diagnosis.

Echocardiography and SPECT are two of the commonly used tools to clinically evaluate myocardial activity. However, due to their limited spatial resolution, these tools only provide an 'all' or 'nothing' result for myocardial activity (14). Notably, even in a single myocardial segment, there may be both viable and unviable parts, for example, the outer membrane of an endocardium with myocardial infarction may be viable. Therefore, the transmural extent of myocardial infarction should be considered segmentally, rather than the activity of a myocardial segment being judged by the concept of 'all' or 
Table I. Extent of coronary artery stenosis and segments with myocardial infarction of different transmural extents detected by DE-MRI.

\begin{tabular}{lrrrrr}
\hline \multirow{2}{*}{$\begin{array}{l}\text { Extent of coronary } \\
\text { artery stenosis (\%) }\end{array}$} & \multicolumn{5}{c}{ Transmural extent of myocardial infarction } \\
\cline { 2 - 5 } & I & II & III & IV & Overall \\
\hline$\leq 25$ & 9 & 4 & 6 & 4 & 23 \\
$\leq 50$ & 19 & 2 & 0 & 2 & 23 \\
$\leq 75$ & 4 & 2 & 2 & 2 & 10 \\
$\leq 90$ & 6 & 12 & 10 & 4 & 10 \\
$\leq 99$ & 2 & 0 & 6 & 12 & 20 \\
$\leq 100$ & 0 & 4 & 4 & 34 & 126 \\
Overall & 40 & 24 & 28 & & 12 \\
\hline
\end{tabular}

DE-MRI, delayed enhancement magnetic resonance imaging. The Kendall rank correlation coefficient was $0.405(\mathrm{P}<0.001)$.
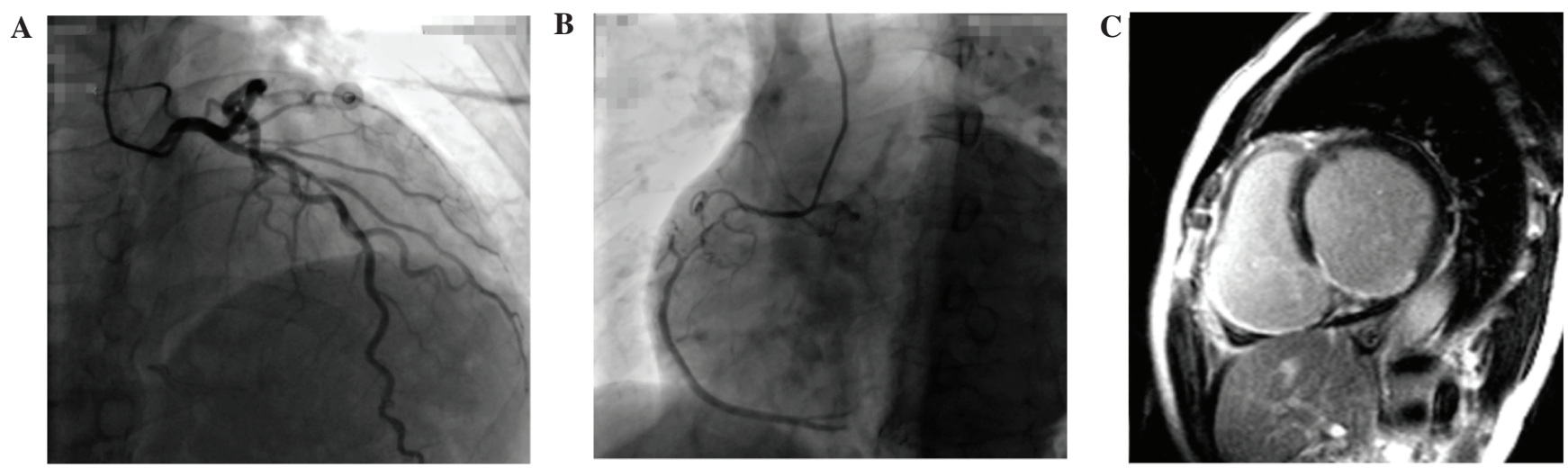

Figure 1. Images of a patient with coronary artery stenosis. Coronary digital subtraction angiography of (A) the left anterior descending coronary artery and (B) the right coronary artery revealed a stenosis extent of 25 and $90 \%$, respectively; (C) Left ventricle partitioning and inferior infarction with a extent of $50-75 \%$ were observed by delayed enhancement magnetic resonance imaging.

A

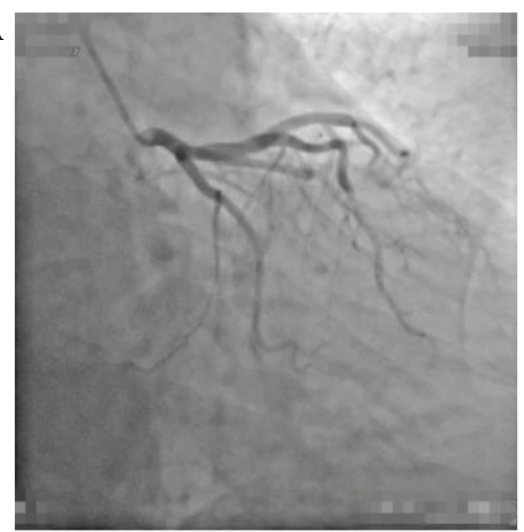

B

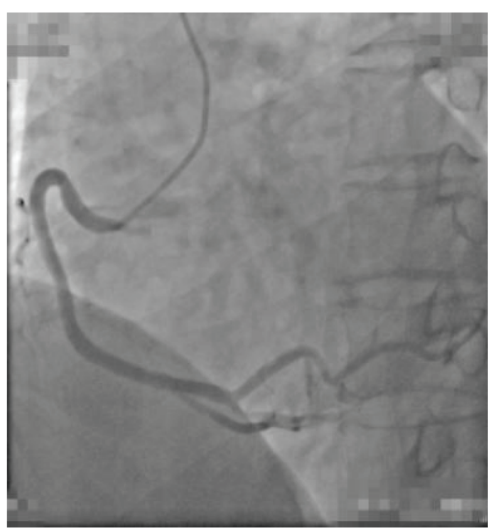

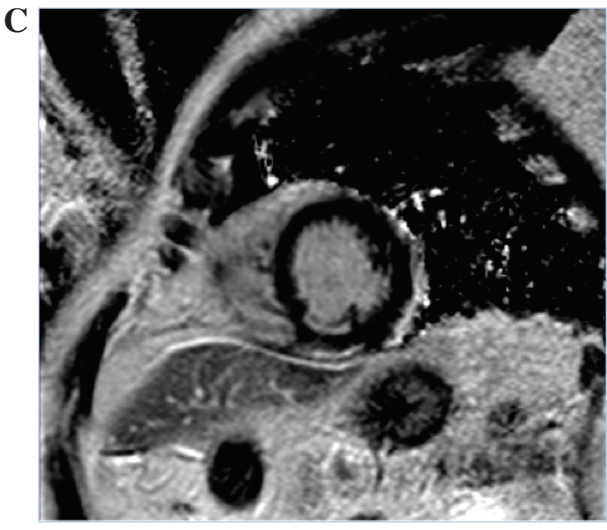

Figure 2. Patient without abnormal signs indicative of coronary artery stenosis. No stenosis was observed in the (A) left anterior descending coronary artery (left circumflex) or (B) right coronary artery using digital subtraction angiography, but (C) delayed enhancement magnetic resonance imaging showed $\leq 25 \%$ infarction extent in the left ventricle, with partitioning and inferior infarction.

'nothing' $(15,16)$. Therefore, analysis of the extent of coronary artery stenosis together with analysis of the transmural extent of myocardial infarction by DE-MRI should benefit both the clinical treatment and prognosis of patients.

The present study showed only a subtle correlation $(\tau=0.405, \mathrm{P}<0.001)$ between the extent of coronary artery stenosis and Kendall rank of the transmural extent of myocardial infarction. It was found that 46 of the segments with myocardial infarction occurred in areas of vascular distribution that exhibited $<50 \%$ coronary artery stenosis. Stenosis $<50 \%$ is unlikely to result in myocardial ischemia due to a compensatory effect in which the resistance of the coronary 
small vasculature is reduced (17). Therefore, $>50 \%$ coronary artery stenosis can be considered as clinically significant for lesions. However, acute coronary syndrome may potentially occur due to plaque rapture in stenosis sites showing $<50 \%$ coronary artery stenosis. In the present study, $18.25 \%$ (23/126) of segments with myocardial infarction were observed in patients without significant coronary artery stenosis $(<25 \%$ stenosis). In addition to plaque rupture and fall off, severe stenosis in coronary spasm may also result in irreversible myocardial damage (18).

Myocardial scarring was found in patients whose coronary artery showed no significant stenosis in a previous study that analyzed the correlation between the severity of coronary artery lesions and DE-MRI results by Bexell et al (19). In addition, the majority of the infarcts were generally subendocardial (with $<25 \%$ transmural extent). The results of the present study were consistent with these data. It was found that $60.87 \%$ of the segments with myocardial infarction showed a transmural extent $<25 \%$ in a coronary artery blood-supplying area in which the extent of coronary stenosis was $<50 \%$. Misdiagnosis may potentially occur if SPECT or positron emission tomography (PET) is used as a diagnosis tool. In a previous study, only $28 \%$ of the segments affected by myocardial infarction were detected by PET compared with $92 \%$ by DE-MRI; this may have been because small areas of scarring could only be detected by DE-MRI (2). Another study with 31 cases of severe heart failure found that $55 \%$ of subendocardial infarction was missed by PET (20). The mortality rate for individuals with undiagnosed myocardial infarction is significantly higher than that for diagnosed myocardial infarction (4). DE-MRI exhibits clear advantages in subendocardial myocardial infarction and small infarcts $(21,22)$.

No evident stenosis was observed by coronary artery DSA in 3 patients. Two of these cases were inferior infarction, mainly subendocardial, with 25-50\% transmural extent (as shown for one patient in Fig. 2). At present, the incidence of coronary artery disease is exhibiting a trend towards younger patients. The patients with no evident stenosis included 2 patients younger than 30 years old, and one was a 26 -year-old female patient. This patient was first diagnosed with inferior wall infarct by DE-MRI, and then right coronary artery stenosis was identified with an extent of $90 \%$ by DSA. Therefore, DE-MRI is helpful in determining the causes of myocardial damage, which are of great significance to prognosis and intervention. A previous study showed a correlation between DE-MRI-detected myocardial infarction and short- or long- term adverse cardiac events, which is also helpful for the evaluation of prognosis (23).

In conclusion, DSA evaluation and DE-MRI may complement each other advantageously, and should not only be helpful for the identification of transmural extent in the viable or irreversible myocardium, but also for the elimination of misdiagnosis.

\section{Acknowledgements}

This study was supported by the National Science Foundation of China (grant no. 81260213), the National Science Foundation of Yunnan Province (grant no. 2012FB054) and Key Projects of Science Research of the Department of Education in Yunnan Province (grant no. 2015HB068).

\section{References}

1. Struyven JJ, Delcour C, Brion JP, Vandenbosch G and Claessens J: Digital subtraction angiography (DSA) of the heart and coronary arteries. Ann Radiol (Paris) 29: 107-111, 1986.

2. Ibrahim T, Bulow HP, Hackl T, Hörnke M, Nekolla SG, Breuer M, Schömig A and Schwaiger M: Diagnostic value of contrast-enhanced magnetic resonance imaging and single-photon emission computed tomography for detection of myocardial necrosis early after acute myocardial infarction. J Am Coll Cardiol 49: 208-216, 2007.

3. Larose E: Below radar: Contributions of cardiac magnetic resonance to the understanding of myonecrosis after percutaneous coronary intervention. Circulation 114: 620-622, 2006.

4. Schelbert EB, Cao JJ, Sigurdsson S, Aspelund T, Kellman P, Aletras AH, Dyke CK, Thorgeirsson G, Eiriksdottir G, Launer LJ, et al: Prevalence and prognosis of unrecognized myocardial infarction determined by cardiac magnetic resonance in older adults. JAMA 308: 890-896, 2012.

5. McManus DD, Chinali M, Saczynski JS, Gore JM, Yarzebski J, Spencer FA, Lessard D and Goldberg RJ: 30-year trends in heart failure in patients hospitalized with acute myocardial infarction. Am J Cardiol 107: 353-359, 2011.

6. Cerqueira MD, Weissman NJ, Dilsizian V, Jacobs AK, Kaul S, Laskey WK, Pennell DJ, Rumberger JA, Ryan T and Verani MS; American Heart Association Writing Group on Myocardial Segmentation and Registration for Cardiac Imaging: Standardized myocardial segmentation and nomenclature for tomographic imaging of the heart. A statement for healthcare professionals from the Cardiac Imaging Committee of the Council on Clinical Cardiology of the American Heart Association. Circulation 105: 539-542, 2002.

7. Gensini GG, Kelly AE, Da Costa BC and Huntington PP: Quantitative angiography: The measurement of coronary vasomobility in the intact animal and man. Chest 60: 522-530, 1971.

8. Okada DR, Liu Z, Johnson G III, Beju D, Khaw BA and Okada RD: ${ }^{99 \mathrm{~m}} \mathrm{Tc}$-glucarate kinetics differentiate normal, stunned, hibernating and nonviable myocardium in a perfused rat heart model. Eur J Nucl Med Mol Imaging 37: 1909-1917, 2010.

9. Ingkanisorn WP, Rhoads KL, Aletras AH, Kellman P and Arai AE: Gadolinium delayed enhancement cardiovascular magnetic resonance correlates with clinical measures of myocardial infarction. J Am Coll Cardiol 43: 2253-2259, 2004.

10. Choi KM, Kim RJ, Gubernikoff G, Vargas JD, Parker M and Judd RM: Transmural extent of acute myocardial infarction predicts long-term improvement in contractile function. Circulation 104: 1101-1107, 2001.

11. Wu E, Judd RM, Vargas JD, Klocke FJ, Bonow RO and Kim RJ: Visualisation of presence, location and transmural extent of healed Q-wave and non-Q-wave myocardial infarction. Lancet 357: 21-28, 2001.

12. Yang J, Ma H, Liu J, Wang C, Shi Y, Xie H, Huo F, Liu F and Lin K: Delayed-enhancement magnetic resonance imaging at $3.0 \mathrm{~T}$ using $0.15 \mathrm{mmol} / \mathrm{kg}$ of contrast agent for the assessment of chronic myocardial infarction. Eur J Radiol 83: 778-782, 2014.

13. Kim RJ, Wu E, Rafael A, Chen EL, Parker MA, Simonetti O, Klocke FJ, Bonow RO and Judd RM: The use of contrast-enhanced magnetic resonance imaging to identify reversible myocardial dysfunction. N Engl J Med 343: 1445-1453, 2000.

14. Baumgartner H, Porenta G, Lau YK, Wutte M, Klaar U, Mehrabi M, Siegel RJ, Czernin J, Laufer G, Sochor H, et al: Assessment of myocardial viability by dobutamine echocardiography, positron emission tomography and thallium-201 SPECT: Correlation with histopathology in explanted hearts. J Am Coll Cardiol 32: 1701-1708, 1998.

15. Prati F, Pawlowski T, Gil R, Labellarte A, Gziut A, Caradonna E, Manzoli A, Pappalardo A, Burzotta F and Boccanelli A: Stenting of culprit lesions in unstable angina leads to a marked reduction in plaque burden: A major role of plaque embolization? A serial intravascular ultrasound study. Circulation 107: 2320-2325, 2003. 
16. Ricciardi MJ, Wu E, Davidson CJ, Choi KM, Klocke FJ, Bonow RO, Judd RM and Kim RJ: Visualization of discrete microinfarction after percutaneous coronary intervention associated with mild creatine kinase-MB elevation. Circulation 103: 2780-2783, 2001.

17. Kuncheng L: China Medical Imagine (cardiovascular system volume). People's Health Publishing House, Beijing, p.88, 2007.

18. Maseri A, L'Abbate A, Baroldi G, Chierchia S, Marzilli M, Ballestra AM, Severi S, Parodi O, Biagini A, Distante A and Pesola A: Coronary vasospasm as a possible cause of myocardial infarction. A conclusion derived from the study of "preinfarction" angina. N Engl J Med 299: 1271-1277, 1978.

19. Bexell D, Setser RM, Schoenhagen P, Lieber ML, Brener SJ, Ivanc TB, Balazs EM,O'Donnell TP, Stillman AE, Arheden H, etal: Influence of coronary artery stenosis severity and coronary collateralization on extent of chronic myocardial scar: Insights from quantitative coronary angiography and delayed-enhancement MRI. Open Cardiovasc Med J 2: 79-86, 2008.

20. Barbier CE, Bjerner T, Johansson L, Lind L and Ahlstrom H: Myocardial scars more frequent than expected: Magnetic resonance imaging detects potential risk group. J Am Coll Cardiol 48: 765-771, 2006.
21. Wagner A, Mahrholdt H, Holly TA, Elliott MD, Regenfus M, Parker M, Klocke FJ, Bonow RO, Kim RJ and Judd RM: Contrast-enhanced MRI and routine single photon emission computed tomography (SPECT) perfusion imaging for detection of subendocardial myocardial infarcts: An imaging study. Lancet 361: 374-379, 2003.

22. Wu YW, Tadamura E, Yamamuro M, Kanao S, Marui A, Tanabara K, Komeda M and Togashi K: Comparison of contrast-enhanced MRI with ${ }^{18} \mathrm{~F}-\mathrm{FDG}$ PET/201T1 SPECT in dysfunctional myocardium: Relation to early functional outcome after surgical revascularization in chronic ischemic heart disease. J Nucl Med 48: 1096-1103, 2007.

23. Kwong RY, Sattar H, Wu H, Vorobiof G, Gandla V, Steel K, Siu S and Brown KA: Incidence and prognostic implication of unrecognized myocardial scar characterized by cardiac magnetic resonance in diabetic patients without clinical evidence of myocardial infarction. Circulation 118: 1011-1020, 2008. 